\title{
Spatial Quantum Beats in Vibrational Resonant Inelastic Soft X-Ray Scattering at Dissociating States in Oxygen
}

\author{
A. Pietzsch, ${ }^{1}$ Y.-P. Sun, ${ }^{2, *}$ F. Hennies, ${ }^{1,3}$ Z. Rinkevicius, ${ }^{2,4}$ H. O. Karlsson, ${ }^{5}$ T. Schmitt, ${ }^{6}$ V. N. Strocov, ${ }^{6}$ J. Andersson, ${ }^{3}$ \\ B. Kennedy, ${ }^{1}$ J. Schlappa, ${ }^{7}$ A. Föhlisch, ${ }^{7,8}$ J.-E. Rubensson, ${ }^{3}$ and F. Gel'mukhanov ${ }^{2}$ \\ ${ }^{1}$ MAX-lab, Lund University, Box 118, S-221 00 Lund, Sweden \\ ${ }^{2}$ Department of Theoretical Chemistry and Biology, School of Biotechnology, Royal Institute of Technology, \\ S-106 91 Stockholm, Sweden \\ ${ }^{3}$ Department of Physics and Astronomy, Uppsala University, Box 516, S-751 20 Uppsala, Sweden \\ ${ }^{4}$ Swedish e-Science Research Center (SeRC), Royal Institute of Technology, SE-100 44 Stockholm, Sweden \\ ${ }^{5}$ Department of Physical and Analytical Chemistry, Uppsala University, Box 518, S-751 20 Uppsala, Sweden \\ ${ }^{6}$ Swiss Light Source, Paul Scherrer Institut, CH-5232 Villigen PSI, Switzerland \\ ${ }^{7}$ Institute for Methods and Instrumentation in Synchrotron Radiation Research G-I2, Helmholtz-Zentrum Berlin für Materialien \\ und Energie, Albert-Einstein-Strasse 15, 12489 Berlin, Germany \\ ${ }^{8}$ Fakultät für Physik und Astronomie, Universität Potsdam, Karl-Liebknecht-Strasse 24-25, 14476 Potsdam, Germany
}

(Received 2 February 2011; published 14 April 2011)

\begin{abstract}
Resonant inelastic soft $x$-ray scattering (RIXS) spectra excited at the $1 \sigma_{g} \rightarrow 3 \sigma_{u}$ resonance in gasphase $\mathrm{O}_{2}$ show excitations due to the nuclear degrees of freedom with up to 35 well-resolved discrete vibronic states and a continuum due to the kinetic energy distribution of the separated atoms. The RIXS profile demonstrates spatial quantum beats caused by two interfering wave packets with different momenta as the atoms separate. Thomson scattering strongly affects both the spectral profile and the scattering anisotropy.
\end{abstract}

DOI: 10.1103/PhysRevLett.106.153004

PACS numbers: 33.20.Rm, 32.80.Aa, 33.20.Fb, 33.80.Gj

Interference is a direct manifestation of the wave nature of matter, most clearly demonstrated in Young's double-slit experiment. When a genuine double-slit experiment is performed with free atoms [1] care must be taken to make an atomic beam with sufficient temporal as well as spatial coherence for interference fringes to be observed. In spectroscopic studies double-slit analogues are encountered, e.g., in the oscillatory time dependence of the decay probability following sudden excitation of two levels of a quantum system, referred to as quantum beats [2]. Spatial quantum beats [3], although conceptually more directly related to the wave nature of matter, have been given much less attention. Here we report on spatial quantum beats in resonant inelastic soft x-ray scattering (RIXS) spectra of the $\mathrm{O}_{2}$ molecule, where two dissociating states form the analogue of the double slit. Initially, the molecule is in the zero-point vibrational level of the electronic ground state. This defines the spatial initial conditions, and also the temporal initial conditions as the excitation process is fast in comparison to the nuclear motion. Depending on which state is excited the atoms separate with different speeds, and thus their phase difference varies with internuclear distance. The resulting interference fringes are monitored in radiative transitions, which project the wave packet development in discrete as well as continuum vibrational excitations of the electronic ground state.

It has long been realized that the decay of core excited states in molecules contains information about the femtosecond nuclear dynamics during the excitationemission process [4]. In the electronic decay channel this feature is currently widely applied, and resonant Auger spectra excited at the same resonance that we use in the present study, the $1 \sigma_{g} \rightarrow 3 \sigma_{u}$ resonance in $\mathrm{O}_{2}$, have been analyzed in detail [5]. Nuclear dynamics has also been addressed in RIXS experiments (see, e.g., Refs [6-9]), in which dipole selection rules imply a high sensitivity to the symmetry of the wave functions, and simplify the interpretation of the spectra. In most studies, however, the energy resolution and counting statistics in the RIXS spectra have been compromised due to experimental limitations, and have not been in parity with what is achievable in resonant Auger spectra. With the new generation of instrumentation this is changing dramatically, and as we have recently demonstrated, individual vibrations can now be readily resolved in molecular RIXS spectra [10].

As in our previous study [10] we have used the SAXES spectrometer [11] at the ADRESS beam line [12] of the Swiss Light Source, Paul Scherrer Institut, Villigen PSI. Gas-phase measurements were facilitated using a flow cell with a $100 \mathrm{~nm}$ thick diamondlike window, separating the ultrahigh vacuum from the sample gas. Incoming and outgoing radiation passed though the same window, both at an angle of $45^{\circ}$.

Scattering at the $1 \sigma_{g} \rightarrow 3 \sigma_{u}$ resonance [Fig. 1(a)] includes a well-resolved vibrational progression with (a) some 35 narrow (FWHM $=50 \mathrm{meV}$, determined by the experimental resolution) peaks in the $0-5 \mathrm{eV}$ energy 

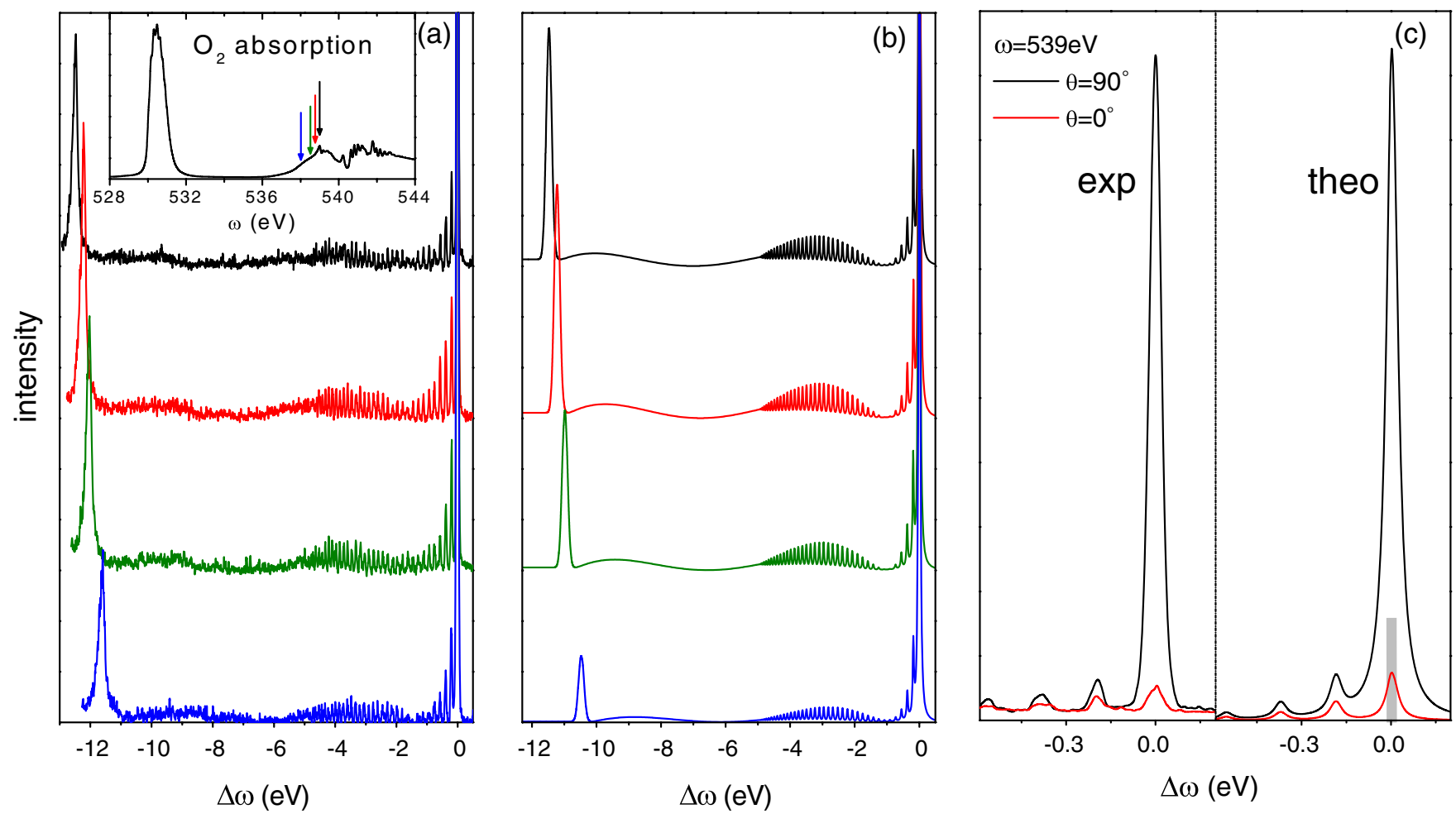

FIG. 1 (color online). Experimental (a) and theoretical (b) RIXS spectra for $\theta=90^{\circ} ; \omega=539,538.75,538.5,538 \mathrm{eV}$ (from top to bottom) as indicated in the absorption spectrum in the top panel. The theoretical "atomic" peak includes also contribution from the dissociative $1^{3} \Pi_{g}$ final state which converges to the same dissociative limit as the ground state. The polarization dependence of the RIXS spectrum shown in panel (c) demonstrates that scattering to $\nu=0$ has a different dependence on the angle $\theta=\angle\left(\mathbf{e}, \mathbf{n}_{1}\right.$ ) (here $\theta=0^{\circ}$ and $90^{\circ}$ ), than scattering to $\nu>0$. The gray bar shows the intensity of the elastic peak intensity for $\theta=90^{\circ}$ without Thomson scattering.

loss range, which transforms into (b) a continuum with smooth intensity variation in the 5-11 eV range, and (c) a sharp Lorentzian $(\mathrm{FWHM} \approx 0.2 \mathrm{eV})$ peak at $11.5-12.5 \mathrm{eV}$ loss. The RIXS spectra are due to transitions to the ${ }^{3} \Sigma_{g}^{-}$ electronic ground state represented by the whole potential curve up to the $O\left({ }^{3} P\right)+O\left({ }^{3} P\right)$ dissociation limit (around $5 \mathrm{eV}$ [13]). The features are assigned to (a) bound vibronic states, (b) losses corresponding to kinetic energy distribution of the separating atoms, and (c) emission from the atoms at full speed. We see an intensity variation of the intensity envelope of the discrete final states with a minimum around $1.5-2 \mathrm{eV}$ and a maximum around $4 \mathrm{eV}$, followed in the continuum by a minimum around $7 \mathrm{eV}$, and a faint maximum around $9 \mathrm{eV}$. The energy position of the atomic peak, finally, equals the kinetic energy of the separated atoms plus the ground state dissociation energy. In the following we will demonstrate that this phenomenology represents spatial quantum beats, due to the excitation of two nonequivalent dissociating intermediate ${ }^{3} \Sigma_{u}^{-}\left(1 \sigma_{g}^{-1} 3 \sigma_{u}\right)$ states. Although this cannot be directly observed in the absorption spectrum it has earlier been demonstrated $[14,15]$ that there are two such states due to two possible internal spin couplings: the spin of the remaining $1 \sigma_{g}$ electron can be parallel or antiparallel to the spin of the two unpaired electrons in the $1 \pi_{g}$ orbital, and the states are denoted $Q$ (for quartet) and $D$ (for doublet), respectively.

Before addressing the dynamics in more detail we outline the numerical $a b$ initio procedure. The simulations of the RIXS spectra shown in Fig. 2 were performed using the time-dependent wave packet technique [16,17]. Potential energy surfaces of the ground and core excited $Q$ and $D$ states in the $0.9<R<2.0 \AA$ region were computed using a complete active space (CAS) wave-function based approach. The $D$ state potential energy surface was determined using the CASSCF/MRPT2 method, in which all 16 electrons of $\mathrm{O}_{2}$ are included in an active space consisting of 10 orbitals used in wave-function optimization, and dynamic electron correlation effects were accounted for using multireference second order perturbation theory (MRPT2). The $Q$ state wave function was obtained with the $D$ state wave function as reference using the configuration interaction method. All calculations were carried out using aug-cc-pCTVZ basis set [18] augmented by midbond basis functions taken from Ref. [15], which are introduced to achieve a better description of the Rydberg states. Core orbital relaxation and static electron correlation effects are treated in a self-consistent fashion allowing for a rigorous 


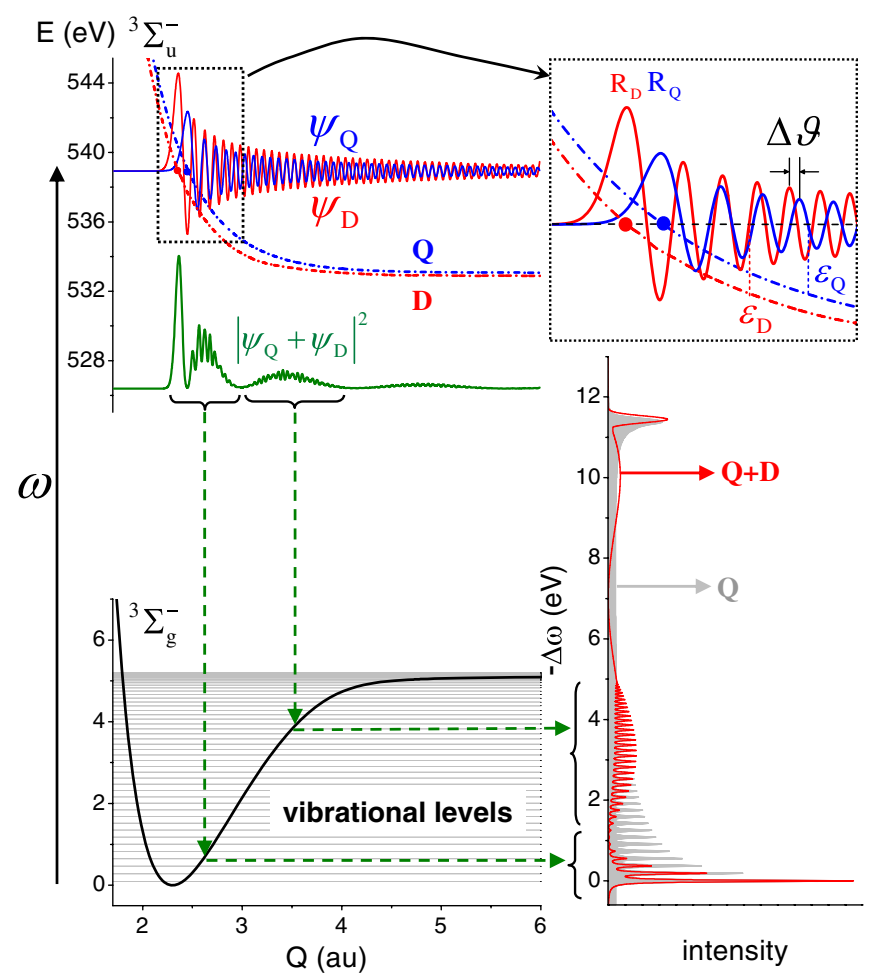

FIG. 2 (color online). Physical picture of the modulations in the vibrational RIXS spectrum. The calculated RIXS spectrum (gray profile in the right panel) with one intermediate state does not display any envelope oscillations and differs qualitatively from the experimental spectrum. The interference between the $Q$ and $D$ scattering channels results in a spatial modulation of the total wave packet $\Psi_{Q}+\Psi_{D}$ which leads to the beat structure in the RIXS spectrum (" $Q+D$ " spectrum in the right panel). The reflection principle explains qualitatively how the spatial modulation of $\left|\Psi_{Q}+\Psi_{D}\right|^{2}$ gives the intensity modulation in the RIXS spectrum.

description of core and valence orbital reorganization upon $1 \sigma_{g} \rightarrow 3 \sigma_{u}$ excitation.

Below we give a simplified physical picture of the spatial beat phenomenon in RIXS. The double differential cross section for vibrational RIXS, as a function of the primary photon energy $\omega$ and energy loss $\Delta \omega=\omega_{1}-\omega$, where $\omega_{1}$ is the energy of the secondary photon, is

$$
\begin{aligned}
\sigma(\omega, \Delta \omega)= & r_{0}^{2} \frac{\omega_{1}}{\omega} \sum_{\nu}\left[\left(\mathbf{e}_{1} \cdot \mathbf{e}\right)^{2}\left(Z^{2}+\frac{2 Z}{3} \operatorname{Re} F_{0}\right) \delta_{\nu, 0}\right. \\
& \left.+\frac{1+2\left(\mathbf{e}_{1} \cdot \mathbf{e}\right)^{2}}{15}\left|F_{\nu}\right|^{2}\right] \Phi\left(\Omega_{\nu}, \gamma\right) .
\end{aligned}
$$

Here $r_{0}=2.82 \times 10^{-13} \mathrm{~cm}$ is the classical radius of the electron, $Z=16$ is the number of electrons in $\mathrm{O}_{2}, \nu$ is the vibrational quantum number of the final state, and $\mathbf{e}$ and $\mathbf{e}_{1}$ are the polarization vectors of the primary and secondary photon, respectively. We use atomic units. The terms in Eq. (1) proportional to $Z^{2}$ and $Z \operatorname{Re} F_{0}$ describe Thomson scattering and the interference between Thomson and resonant channels, respectively, while the last term shows the resonant scattering. As the polarization of the secondary photon is not detected, and as the angle between $\mathbf{e}$ and the propagation direction of the secondary photon, $\mathbf{n}_{1}$, is $\theta=\angle\left(\mathbf{e}, \mathbf{n}_{1}\right)=0^{\circ}$ and $90^{\circ},\left(\mathbf{e}_{1} \cdot \mathbf{e}\right)^{2} \rightarrow \frac{1}{2} \sin ^{2} \theta$ in the present experiment. The scattering is on resonance with the two intermediate $Q$ and $D$ dissociative states which have an energy spacing near the ground state equilibrium distance of $\Delta=E_{Q}(R)-E_{D}(R)$. Therefore the scattering amplitude is expressed as the sum $F_{\nu}=F_{\nu}^{D}+F_{\nu}^{Q}$, where the scattering amplitude of the $j$ th channel is the integral over the energy of the dissociative state $\epsilon$ relative to the energy of the vertical transition $\omega_{j 0}$ :

$$
\begin{aligned}
F_{\nu}^{j} & =\omega \omega_{1} d_{j 0}^{2}\left\langle\nu \mid \Psi_{j}\right\rangle, \\
\Psi_{j}(R) & =\int d \epsilon \frac{\left\langle\phi_{j, \epsilon} \mid 0\right\rangle \phi_{j, \epsilon}(R)}{\Omega_{j}-\epsilon+\imath \Gamma} \\
& \approx-\frac{\sqrt{\pi}}{S \sqrt{2}} e^{-\Omega_{j}^{2} / 2 S^{2}}\left[\imath-\sqrt{\frac{2}{\pi}} \frac{\Omega_{j}}{S}\right] \phi_{j, \Omega_{j}+l \Gamma}(R) .
\end{aligned}
$$

Here $d_{j 0}$ is the matrix element for the electronic transition between the ground and intermediate states, which we assume to be independent of the internuclear distance, $\Omega_{j} \equiv \omega-\omega_{j 0}$ is the detuning of $\omega$ relative to $\omega_{j 0}$, and $S$ is the spectral width of the Franck Condon factor $\left\langle\phi_{j, \epsilon} \mid 0\right\rangle^{2}$. Integrating over $\epsilon$ we use that $\Gamma \ll S$ so that the main contribution to the integral $\left\langle\phi_{j, \epsilon} \mid 0\right\rangle$ comes from the pole $\epsilon=\Omega_{j}+\imath \Gamma$.

Both wave packets propagate along the energy surface of the intermediate states with the total energy $\omega$ (see Fig. 2). When the nuclei separate the kinetic energy of the wave packets increases as the potential decreases. Two different potential surfaces imply two different kinetic energies $\varepsilon_{j}(R)$ and hence, different wave numbers $k_{j}(R)=$ $\sqrt{2 \mu \varepsilon_{j}(R)}$ and the related velocity $v_{j}(R)=k_{j}(R) / \mu$, where $\mu$ is the reduced mass of the atoms. As shown in Eq. (2) the phase of the wave packet $\Psi_{j}(R)$ is the same as the phase of the nuclear wave function $\phi_{j \Omega_{j}}(R)$. The semiclassical expression for the nuclear wave function results in

$$
\Psi_{j}(R) \propto \exp \left(\int_{R_{j}}^{R}\left[\imath k_{j}\left(R_{1}\right) d R_{1}-\Gamma d \tau\right]\right), \quad d \tau=\frac{d R_{1}}{v_{j}\left(R_{1}\right)} .
$$

Now we can estimate the phase difference $\Delta \vartheta$ between the $Q$ and $D$ wave packets

$$
\Delta \vartheta+\varphi \approx\left(k_{Q}-k_{D}\right)\left(R-R_{Q}\right)=-\tau \Delta,
$$

where $\tau=\left(R-R_{Q}\right) / v$ is the time of the propagation of the wave packet to the point $R$ with the averaged velocity $v=\left(k_{Q}+k_{D}\right) / 2 \mu$ and $\varphi$ is the constant phase shift between the $Q$ and $D$ wave packets caused by the spacing between the classical turning points $R_{Q}-R_{D}$ (see Fig. 2). 
The changing phase difference results in a slow spatial modulation of the total wave packet $\Psi(R)=\Psi_{D}(R)+$ $\Psi_{Q}(R)$ which is seen in Fig. 2. At increasing $R$ the beating (Fig. 2) is damped due to the decay of the core excited state:

$$
\cos (\tau \Delta+\varphi) e^{-2 \Gamma \tau}
$$

The number of oscillations for continuum-continuum transitions is very sensitive to the $Q D$ splitting $\Delta$. For example, we get two oscillations instead of one seen in the experiment if we use the value $\Delta \approx 2 \mathrm{eV}$ calculated earlier $[5,14,15]$. The splitting (near the equilibrium) calculated in the present article $\Delta \approx 1.4 \mathrm{eV}$ gives only one modulation which is consistent with the experiment (Fig. 2). The atomic peak is computed assuming that the $Q$ and $D$ core excited states converge to the same dissociative limit $O\left({ }^{3} P\right)+O^{*}\left({ }^{3} P\right)$.

Finally, we would like to point out that Thomson scattering is the dominant channel for the elastic $(\nu=0)$ peak, as the first term in Eq. (1) gives a much larger contribution to the intensity than the second. The reason for this is the strong suppression $(\Gamma / S \approx 0.05)$ of the resonant term caused by the dissociative nature of the core excited state. Thomson scattering influences only the final vibrational state $\nu=0$, and it has an angular dependence which is different from the resonant term. In contrast to resonant scattering, Thomson scattering is absent for parallel geometry, $\theta=0$. Thus, the vibrational progression is anisotropic. By comparing spectra measured in parallel $(\theta=0)$ and perpendicular $\left(\theta=90^{\circ}\right)$ geometry [see Fig. 1(c)] we demonstrate that the cross section of Thomson scattering is about 7 times larger than the cross section for resonant scattering.

In conclusion, we have observed the spatial quantum beat effect in vibrational RIXS of molecular oxygen. Our study shows that the beat structure is very sensitive to the splitting between two close-lying dissociative core excited states. In addition, the combined experimental and theoretical study demonstrates the importance of the Thomson channel in scattering via dissociative core excited states.

This work was performed at the ADRESS beam line of the Swiss Light Source using the SAXES instrument jointly built by Paul Scherrer Institut, Switzerland and Politecnico di Milano, Italy. We acknowledge support by the Swedish Research Council (VR) and Carl Tryggers Stiftelse (CTS). *yuping@ theochem.kth.se

[1] O. Carnal and J. Mlynek, Phys. Rev. Lett. 66, 2689 (1991).

[2] G. C. Hegerfeldt and M. B. Plenio, Quantum Opt. 6, 15 (1994).

[3] Z. Y. Ou and L. Mandel, Phys. Rev. Lett. 61, 54 (1988).

[4] T.X. Carroll, S.E. Anderson, L. Ungier, and T.D. Thomas, Phys. Rev. Lett. 58, 867 (1987).

[5] R. Feifel, Y. Velkov, V. Carravetta, C. Angeli, R. Cimiraglia, P. Sałek, F. Gel'mukhanov, S. L. Sorensen, M. N. Piancaśtelli, A. De Fanis, K. Okada, M. Kitajima, T. Tanaka, H. Tanaka, and K. Ueda, J. Chem. Phys. 128, 064304 (2008).

[6] P. Skytt, P. Glans, J.-H. Guo, K. Gunnelin, C. Såthe, J. Nordgren, F. K. Gel'mukhanov, A. Cesar, and H. Ågren, Phys. Rev. Lett. 77, 5035 (1996).

[7] M. Simon, L. Journel, R. Guillemin, W. C. Stolte, I. Minkov, F. Gel'mukhanov, P. Sałek, H. Ågren, S. Carniato, R. Taïeb, A.C. Hudson, and D.W. Lindle, Phys. Rev. A 73, 020706 (2006).

[8] C. Såthe, F. F. Guimarães, J.-E. Rubensson, J. Nordgren, A. Agui, J. Guo, U. Ekström, P. Norman, F. Gel'mukhanov, and H. Ågren, Phys. Rev. A 74, 062512 (2006).

[9] F. Hennies, S. Polyutov, I. Minkov, A. Pietzsch, M. Nagasono, F. Gel'mukhanov, L. Triguero, M.-N. Piancastelli, W. Wurth, H. Ågren, and A. Föhlisch, Phys. Rev. Lett. 95, 163002 (2005).

[10] F. Hennies, A. Pietzsch, M. Berglund, A. Föhlisch, T. Schmitt, V. Strocov, H. O. Karlsson, J. Andersson, and J.-E. Rubensson, Phys. Rev. Lett. 104, 193002 (2010).

[11] G. Ghiringhelli, A. Piazzalunga, C. Dallera, G. Trezzi, L. Braicovich, T. Schmitt, V. N. Strocov, R. Betemps, L. Patthey, X. Wang, and M. Grioni, Rev. Sci. Instrum. 77, 113108 (2006).

[12] V. N. Strocov, T. Schmitt, U. Flechsig, T. Schmidt, A. Imhof, Q. Chen, J. Raabe, R. Betemps, D. Zimoch, J. Krempasky, X. Wang, M. Grioni, A. Piazzalunga, and L. Patthey, J. Synchrotron Radiat. 17, 631 (2010).

[13] F. R. Gilmore, J. Quant. Spectrosc. Radiat. Transfer 5, 369 (1965).

[14] J. Adachi, N. Kosugi, and A. Yagishita, J. Phys. B 38, R127 (2005).

[15] I. Hjelte, O. Björneholm, V. Carravetta, C. Angeli, R. Cimiraglia, K. Wiesner, S. Svensson, and M. N. Piancastelli, J. Chem. Phys. 123, 064314 (2005).

[16] P. Sałek, F. Gel'mukhanov, and H. Ågren, Phys. Rev. A 59, 1147 (1999).

[17] F. Gel'mukhanov and H. Ågren, Phys. Rep. 312, 87 (1999).

[18] T. H. Dunning Jr., J. Chem. Phys. 90, 1007 (1989). 